\title{
THE CONDITION FOR AN APPROXIMATION OF POISSON DISTRIBUTION TO BERNOULLI SUMS IN MULTIVARIATE DISTRIBUTION
}

\author{
By KaZUTOMO KaWAMURA
}

\section{$\S 1$. Summary.}

K. Kawamura [1] has discussed that a condition is shown and it plays as sufficient condition for an approximation of Poisson distribution to the sum of Bernoulli sequences and he has investigated the structure of Poisson distribution in multivariate case. C. Liu [2] also has discussed an approximation to the sum of variable (non-identically distributed) Bernoulli sequences.

In this paper the converse assertion is discussed, that is, the condition is essential for the approximation of Poisson distribution to the sum of independent Bernoulli sequences in multivariate case. The notations and discussion will prepare the break through in the case of variable Bernoulli sequences.

\section{$\S 2$. Notations and definitions.}

$k=\left(k_{1}, k_{2}, \cdots, k_{n}\right)$

where coordinates $k_{j}(j=1,2, \cdots, n)$ are non-negative integers,

$0=(0,0, \cdots, 0)$; zero-vector,

$E_{0}=\{0,1\}^{n}, \quad E=\{0,1\}^{n}-0, \quad i \in E_{0}$,

$\# k$; the number of positive coordinates in a vector $k$.

An ordering for $i \in E_{0}$ in 3-dimensional case $(n=3)$;

$$
i=\left(\begin{array}{l}
(0,0,0)=000 \\
(1,0,0)=100 \\
(0,1,0)=010 \\
(0,0,1)=001 \\
(1,1,0)=110 \\
(1,0,1)=101 \\
(0,1,1)=011 \\
(1,1,1)=111
\end{array} \quad \# i=0,\right.
$$

Received December 25, 1987 
We use the ordering for $i \in E_{0}$, like above, in multivariate case, and also the ordering for $i \in E$.

$P(\lambda): \quad n$-variate Poisson distribution

$\lambda=\left(\lambda_{10 \ldots 0}, \lambda_{010 \ldots 0}, \cdots, \lambda_{0 \ldots 01}, \cdots, \lambda_{11 \cdots 1}\right): 2^{n}-1$ dimensional parameter each coordinate $\lambda_{k}$ is non-negative parameter where suffix vectors $k=100 \cdots 0,010 \cdots 0, \cdots$, $00 \cdots 01, \cdots, 11 \cdots 1$ are ordered by a given ordering like above (2.1). And also we put

$p=\left(p_{00 \ldots 0}, p_{10 \ldots 0}, p_{010 \ldots 0}, \cdots, p_{0 \ldots 01}, \cdots, p_{11 \ldots 1}\right): 2^{n}$-dimensional vector.

\section{§3. Main result.}

THEOREM 1. For given independent Bernoulli sequences $X_{1}, X_{2}, \cdots, X_{N}$ each having a distribution $B(1, p)$, the sum $X=\sum_{j=1}^{N} X_{j}$ has a binomial distribution $B(N, p)$. The distribution is expressed for $k \in\{0,1, \cdots, N\}^{n}$

$$
P(X=k)=\sum_{\left[C_{0}\right]} \frac{N !}{\prod_{i \in E_{0}} \alpha_{i} !} \prod_{i \in E_{0}} p_{i}^{\alpha_{i}} .
$$

Proof. See Kawamura [1].

THEOREM 2. Let $X$ be a binomial distribution $B(N, p)$ then we have for any $k \geqq 0$

$$
\lim _{\substack{N \rightarrow \infty^{\infty} \\ N p_{i} \rightarrow \lambda_{i}, i \in E}} P(X=k)=\sum_{[C]} \prod_{i \in E} p\left(\alpha_{i} ; \lambda_{i}\right)
$$

where $p(\alpha ; \lambda)$ is an univariate Poisson probability density.

Proof. See Kawamura [1].

In this paper we will show the fact that the limiting condition

$$
\text { " } N p_{i} \longrightarrow \lambda_{i} \text { as } N \longrightarrow \infty \quad \text { for } i \in E \text { " }
$$

is essential to the approximation.

Theorem 3. For given independent Bernoulli sequence $X_{1}, X_{2}, \cdots, X_{N}$ each having a distribution $B(1, p)$ if we assume that the sum $X=\sum X_{j}$ has the property of Poisson approximation:

$$
\lim _{N \rightarrow \infty} P(X=k)=\sum_{[C]} \prod_{i \in E} p\left(\alpha_{i} ; \lambda_{i}\right) \quad(k \geqq 0)
$$

then we can derive the condition

$$
\text { "Np} p_{i} \longrightarrow \lambda_{i} \text { as } N \longrightarrow \infty \quad \text { for } i \in E \text { " }
$$

Proof. Step 1) Defining $\# v$ is a number of positive components in the 
vector $v$ (usually components of $v$ are nonnegative integers).

If $\# k=0$ then $k=0=(0,0, \cdots, 0)$. Put $k=0$ then in the left side of limiting equation (3.3) becomes

$$
P(X=0)=\sum_{\left[C_{0}\right]} \frac{N !}{\prod_{i \in E_{0}} \alpha_{i} !} \prod_{i \in E_{0}} p_{i}^{\alpha}=p_{0}^{N} .
$$

Solution of $\left[C_{0}\right]$ with $k=0$ is simply expressed

for $i \in E_{0}$, then we get

$$
\alpha_{i}=\left[\begin{array}{ll}
N, & i=0, \\
0, & i \neq 0
\end{array}\right.
$$

$$
P(X=O)=p_{o}^{N}
$$

and left side of (3.3) becomes

$$
\lim _{N \rightarrow \infty} P(X=O)=\lim _{N \rightarrow \infty} p_{o}^{N} .
$$

Solution of $[C]$ with $k=0$ is also expressed

$$
\alpha_{i}=0, \quad \text { for } i \in E
$$

then right side of (3.3) becomes

$$
\sum_{[C]} \prod_{i \in E} p\left(\alpha_{i} ; \lambda_{i}\right)=\prod_{i \in E} p\left(0 ; \lambda_{i}\right)=\exp \left\{-\sum_{i \in E} \lambda_{i}\right\}
$$

Therefore, we get

$$
\lim _{N \rightarrow \infty} p_{o}^{N}=\exp \left\{-\sum_{i \in E} \lambda_{i}\right\}
$$

where

$$
\begin{gathered}
p_{0}=1-\sum_{i \in E} p_{i} \\
\lim _{N \rightarrow \infty}\left\{1-\frac{N \sum p_{i}}{N}\right\}^{N}=\exp \left\{-\sum_{i \in E} \lambda_{i}\right\} .
\end{gathered}
$$

Then we can conclude

$$
\lim _{N \rightarrow \infty} N \sum_{i \in E} p_{i}=\lim \sum_{i \in E} N p_{i}=\sum_{i \in E} \lambda_{i}
$$

LEMMA. Under the condition of theorem 3, we have

$$
\lim _{N \rightarrow \infty} N \sum_{i \in E} p_{i}=\sum_{i \in E} \lambda_{i} \text {. }
$$

Step 2) In the case of $k=(100 \cdots 0)$ the solution of $\left[C_{0}\right]$ with $k=(100 \cdots 0)$ is

$$
\begin{aligned}
& \alpha_{o}=N-1, \\
& \alpha_{k}=1,
\end{aligned}
$$




$$
\alpha_{\imath}=0, \quad i \neq 0, k \quad \text { for } i \in E_{0} .
$$

The solution of [C] is $\alpha_{k}=1$ and $\alpha_{i}=0$ for $i \neq k, i \in E$ then from (3.3)

$$
\lim _{N \rightarrow \infty} N p_{k} p_{o}^{N-1}=p\left(1 ; \lambda_{k}\right) \prod_{i \in E, i \neq k} p\left(0 ; \lambda_{i}\right)
$$

then

$$
\lim _{N \rightarrow \infty} N p_{k} \lim _{N \rightarrow \infty} p_{o}^{N-1}=\lambda_{k} \prod_{i \in E} \exp \left\{-\lambda_{i}\right\}
$$

and using (3.6) we can get

$$
\lim _{N \rightarrow \infty} N p_{k}=\lambda_{k} .
$$

In the same way, we get (3.7) for any $k$ satisfying $\# k=1$; that is, under the condition of the theorem if $k \in E$ and $\# k=1$ then we have

$$
\lim _{N \rightarrow \infty} N p_{k}=\lambda_{k} \text {. }
$$

Step 3) Let us proceed to prove the conclusion (3.4) of theorem 3 that for any $k \in E$ we have

$$
\lim _{N \rightarrow \infty} N p_{k}=\lambda_{k},
$$

by the induction of the number of positive components of $k \in E: \# k=r(1 \leqq r \leqq n)$. In step 2 we have proved that the conclusion of the theorem is valid for $r=1$.

HYPOTHESIS OF THE INDUCTION. If we assume the conclusion also valid for every $r: r \leqq r_{0}$ where $1 \leqq r_{0} \leqq n$, then we can prove the relation of (3.7) for $r=$ $r_{0}+1$, as follows and finish the induction.

Put $k \in E, \# k=r_{0}+1$ then if we have to decompcse the vector $k$ as following

$$
k=j_{1}+j_{2}+\cdots+j_{s}
$$

where $j_{1}, j_{2}, \cdots, j_{s}$ are $n$-dimensional vectors and havirg nonnegative integral components, so $j_{1}, j_{2}, \cdots, j_{s} \geqq 0$ and $j_{1}, j_{2}, \cdots, j_{s} \neq 0$ are satisfied.

Let us define the relation of vectors $V$ and $O: V>O \Leftrightarrow V \geqq O$ and $V \neq O$, then the decomposed vectors must satisfy $j_{1}, j_{2}, \cdots, j_{s}>0$. If the vector $k$ in the left side of (3.3) is the sum of $N$ independent Bernoulli vectors havirg a distribution $B(1, p)$ then we express a sequence of $N$ observatiors:

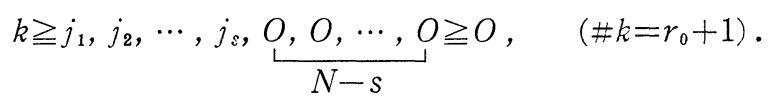

That is a decomposition of $k$ is given by

$$
k=j_{1}+j_{2}+\cdots+j_{s}+\underbrace{O+O+\cdots+O}_{N-s} .
$$

The probability of an occurrence of the decomposition is 


$$
p_{j_{1}} p_{j_{2}} \cdots p_{j_{s}} \underbrace{p_{o} \cdots p_{o}}_{N-s} .
$$

The combination for the decomposition satisfying (3.9) is

$$
\frac{N !}{1 ! 1 ! \cdots 1 !(N-s) !}=N(N-1) \cdots(N-s+1) \text {. }
$$

Therefore the probability of the decomposition having $j_{1}, j_{2}, \cdots, j_{s}>O$ becomes

$$
N(N-1) \cdots(N-s+1) p_{j_{1}} p_{j_{2}} \cdots p_{j_{s}} p_{o}^{N-s} .
$$

To calculate $P(X=k)$ of (3.3) we have to summarize such probabilities for all decompositions satisfying (3.9).

$$
\sum_{s=1}^{r+1} \sum_{\substack{j_{1}+j_{2}+\cdots+j_{s}=k, 0<j_{1}, j_{2}, \cdots, j_{s} \leq 1}} N(N-1) \cdots(N-s+1) p_{j_{1}} p_{j_{2}} \cdots p_{j_{s}} p_{o}^{N-s}
$$

where $O=(0,0, \cdots, 0)$ and $1=(1,1, \cdots, 1)$ are $n$-dimensional vectors. And from the condition (3.3) of the theorem, the fact (3.6) in the lemma is already proved:

$$
\lim _{N \rightarrow \infty} p_{o}^{N}=\exp \left\{-\sum_{i \in E} \lambda_{i}\right\}
$$

and the hypothesis of the induction we have

$$
\begin{aligned}
\lim _{N \rightarrow \infty} P(X=k) & =\sum_{s=1}^{r+1} \Sigma \lim N p_{j_{1}}(N-1) p_{j_{2}} \cdots(N-s+1) p_{j_{s}} \lim p_{o}^{N-s} \\
& =\lim N p_{k} \lim p_{o}^{N-1}+\sum_{s=2}^{r+1} \Sigma \lambda_{j_{1}} \lambda_{j_{2}} \cdots \lambda_{j_{s}} \exp \left\{-\sum_{i \in E} \lambda_{i}\right\} \\
& =\left[\lim N p_{k}+\sum_{s=2}^{r+1} \sum \lambda_{j_{1}} \lambda_{j_{2}} \cdots \lambda_{j_{s}}\right] \exp \left\{-\sum_{i \in E} \lambda_{i}\right\} .
\end{aligned}
$$

On the other hand, in the right side of (3.3) the solution of [C] with $\# k=r_{0}+1$ is expressed

where

$$
\alpha_{j_{1}}=\alpha_{j_{2}}=\cdots=\alpha_{j_{s}}=1 \text { and } \alpha_{i}=0 \text { for } i \neq j_{1}, j_{2}, \cdots, j_{s}
$$

$$
j_{1}+j_{2}+\cdots+j_{s}=k \text { and } 0<j_{1}, j_{2}, \cdots, j_{s} \leqq 1 .
$$

And $\alpha_{j_{1}}, \alpha_{j_{2}}, \cdots, \alpha_{j_{s}}$ are consist with the numbers of the decomposed vectors. $j_{1}, j_{2}, \cdots, j_{s}$ in (3.8). In the right side of (3.3)

$$
\sum_{[C]} \prod_{i \in E} p\left(\alpha_{i} ; \lambda_{i}\right)
$$

the solution of $[C]$ is depending on the decomposition and we have $\alpha_{i}=0$ or 1 from $k \in E$. We have $\alpha_{i}=1$ if $i \in\left\{j_{1}, j_{2}, \cdots, j_{s}\right\}$ of the decomposition and otherwise $\alpha_{i}=0$ for $i \in E$. And we can check from (3.8) and $k \in E$ the decomposed vectors $j_{1}, j_{2}, \cdots, j_{s}$ are mutually different vectors. 


$$
\begin{aligned}
P(X=k) & =\sum_{[C]} \prod_{i=1}^{s} p\left(\alpha_{j_{i}} ; \lambda_{j_{i}}\right) \underset{i \in E, i \neq j_{1}, j_{2}, \cdots, j_{s}}{ } p\left(0 ; \lambda_{i}\right) \\
& =\sum_{s=1}^{\# k} \sum_{\operatorname{Dec}} \prod_{i=1}^{s} \lambda_{j_{i}} \exp \left\{-\lambda_{j_{i}}\right\} \prod_{i \in E, i \neq j_{1}, j_{2}, \cdots, j_{s}} \exp \left\{-\lambda_{i}\right\}
\end{aligned}
$$

where

$$
\begin{aligned}
\operatorname{Dec} & =\left\{j_{1}, j_{2}, \cdots, j_{s}: j_{1}+j_{2}+\cdots+j_{s}=k, 0<j_{i} \leqq 1 \text { and } 0<j_{i} \leqq k\right\} \\
& =\sum_{s=1}^{\# k} \sum_{\operatorname{Lec}} \prod_{i=1}^{s} \lambda_{j_{i}} \prod_{i \in E} \exp \left\{-\lambda_{i}\right\} \\
& =\lambda_{k} \exp \left\{-\sum_{i \in E} \lambda_{i}\right\}+\sum_{s=2}^{\# k} \sum_{\operatorname{Dec}} \prod_{i=1}^{s} \lambda_{j_{i}} \exp \left\{-\sum_{i \in E} \lambda_{i}\right\}
\end{aligned}
$$

Finally we can conclude by (3.11) and (3.12)

$$
\lim _{N \rightarrow \infty} N p_{k}=\lambda_{k} \quad \text { for } \# k=r+1 .
$$

Now, we have finished the induction: the proof of the validity of (3.7) in the theorem for every $k$ satisfying $\# k=r+1$. So we can conclude for any $k: k \in E$ and $\# k=1,2, \cdots, n$

$$
\lim _{N \rightarrow \infty} N p_{k}=\lambda_{k} .
$$

This is the conclusion of the theorem.

These theorems have a variation theorem rather a mathematical one, that is, summarizing Theorem 2 and 3 we conclude next theorem.

THEOREM 4. Necessary and sufficient condition for the convergence of p.g.f. of $B(N, p)$ to p.g.f. of $P(\lambda)$ :

$$
\lim _{N \rightarrow \infty}\left(\sum_{i \in E_{0}} p_{i} s^{i}\right)^{N}=\prod_{i \in E} \exp \left\{-\lambda_{i}+\lambda_{\imath} s^{i}\right\}
$$

is

$$
\lim _{N \rightarrow \infty} N p_{i}=\lambda_{i} \quad \text { for } i \in E .
$$

That is the condition " $\lim _{N \rightarrow \infty} N p_{i}=\lambda_{i}$ for $i \in E$ " (3.4) is essential for the convergence of distribution.

\section{$\S 4$. Bivariate case.}

Let $X_{1}, X_{2}, \cdots, X_{N}$ be a sequence of independent Bernoulli distribution $B(1, p)$ where $p=\left(p_{00} p_{10} p_{01} p_{11}\right), p_{i j} \geqq 0$ and $\sum p_{i j}=1\left(\sum_{i \in E_{0}} p_{i}=1\right)$

$$
P\left(X_{j}=i\right)=p_{i}, \quad i \in E_{0}, \quad \text { for } j=1,2, \cdots, N .
$$

Then from theorem 1 we have 


$$
P\left(\sum_{j=1}^{N} X_{j}=k\right)=\sum_{\substack{\beta+\delta=k_{1} \\ \gamma+\delta=k_{2} \\ \alpha+\beta+\gamma+\delta=N \\ \alpha, \beta, \gamma, \delta \geq 0 \text { integer }}} \frac{N !}{\alpha ! \beta ! \gamma ! \delta !} p_{00}{ }^{\alpha} p_{10}{ }^{\beta} p_{01}{ }^{\gamma} p_{11}{ }^{\delta}
$$

where $k=\left(k_{1}, k_{2}\right) \geqq O: k_{1}, k_{2} \geqq 0$.

Theorem 5. Let $X_{1}, X_{2}, \cdots, X_{N}$ be an independent Bernoulli sequence then we have

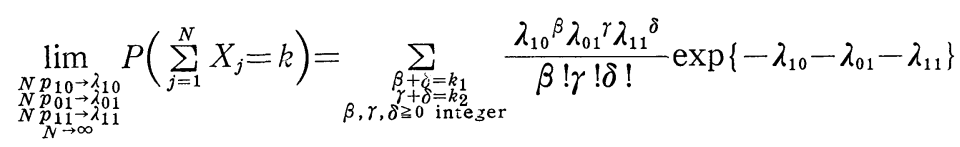

for every $k=\left(k_{1}, k_{2}\right) \geqq 0$.

Proof. See theorem 2.

THEOREM 6. Let $X_{1}, X_{2}, \cdots, X_{N}$ be an independent bivariate Bernoulli sequence and we assume

$$
\lim _{N \rightarrow \infty} P\left(\sum_{j=1}^{N} X_{J}=k\right)=\sum_{\substack{\beta+\delta=k 1 \\ \gamma+\delta=k 2 \\ \beta, \gamma, \delta \geq 0 \text { integer }}} \frac{\lambda_{10}{ }^{\beta} \lambda_{01}^{\gamma} \lambda_{11}{ }^{\delta}}{\beta ! \gamma ! \delta !} \exp \left\{-\lambda_{10}-\lambda_{01}-\lambda_{11}\right\}
$$

then we have $N p_{10} \rightarrow \lambda_{10}, N p_{01} \rightarrow \lambda_{01}$ and $N p_{11} \rightarrow \lambda_{11}$ as $N \rightarrow \infty$.

Proof. See theorem 3.

Acknowledgement. The author expresses his hearty thanks to Cheng-Gee Liu for typing of this paper by personal computer PC-9801 VX2.

\section{REFERENCES}

[1] K. Kawamura, The structure of multivariate Poisson distribution, Kodai Math. J. 2(3) (1979), 337-345.

[2] C. LiU, A note on Poisson approximation in multivariate case, Kodai Math. J. 10 (2) (1987), 223-230.

[3] A. W. Marshall and I. OlKin, A family of bivariate distributions generated by the bivariate Bernoulli distribution, Journal of the A.S. A., (June 1985), 332-338.

Department of Mathematics

Tokyo Institute of Technology

OH-OKAYAMA, MEgURO-KU

TOKYO 152, JAPAN 